\title{
Temporal Compressive Sensing Instrumentation for TEM
}

\author{
Daniel J. Masiel ${ }^{1}$, Ruth S. Bloom ${ }^{1}$, Sang Tae Park ${ }^{1}$ and Bryan W. Reed ${ }^{1}$ \\ 1. Integrated Dynamic Electron Solutions, Inc, Pleasanton United States of America
}

Advances in compressive sensing (CS) techniques and instrumentation have created a renewed interest in exploring new methods for data collection and post-processing [1,2]. Recently developed Temporal CS (TCS) techniques based on post-specimen, high-speed electrostatic beam deflectors effectively multiply the frame rate of commonly available TEM cameras by pre-compressing video data on the detector prior to readout, enabling much higher frame rates for in situ TEM measurements. In addition to improving camera frame rate, TCS opens up a very powerful set of techniques for electron imaging, diffraction, and spectroscopy by mapping time to other external experimental parameters such as probe position, probe strobe frequency, sample orientation, or sample drift. The TCS system introduced here is capable of precisely modulating or recording these experimental parameters through the same Integrated Timing Unit (ITU) that manages the camera and deflector.

The electrostatic beam deflectors employed in this TCS system are mounted on standard TEM peripheral ports (Figure 1) and are capable of $\mathrm{MHz}$ state transitions, at full duty cycle, with 20 ns transit time between frames. This enables them to project the beam onto a $3 \times 3$ or $4 \times 4$ array of sub-frames on the camera in a pseudo random sequence, known as a temporal mask. Each of these sub-frames is exposed multiple times during a sequence, such that each sub-frame image is composed of different combinations of slices in time. A video can then be reconstructed from these multiple exposures because the control system (Figure 2) has precise knowledge of the transition time and sub-frame location of each time slice. Depending on the compressibility of the data, this typically results in $\sim 10-100$ frames of video per camera readout, making kilohertz frame rates feasible for many common TEM cameras.

The user is guided through the reconstruction process by a software package that allows them to adjust the target compression ratio. The software combines the timing data from the ITU as well as the image data from the camera. This includes transition times for each time slice of the acquisition, which is typically triggered by the camera clock or shutter output. The ITU is capable of triggering or recording data from other microscope accessories and sub-systems and integrating that information into the software reconstruction process. The image data can be loaded into the software in a variety of formats and can be customized to function within a given user's data workflow.

The TCS system presented here combines high performance electrostatic charged-particle optics and precision timing systems with statistically meaningful image and video processing algorithms. The outcome is a simple to operate, plug-in microscope accessory that enhances camera frame rate performance; enables timing and control of accessories and microscope sub-systems and thereby completely new operating modes; and pulls information from major components of a user's experiment to enhance their analytical workflow and derive more meaning from their data. 


\section{References:}

[1] B W Reed et al, Microsc. Microanal. (2016) submitted

[2] A. Stevens et al., Adv. Struct. Chem. Imaging (2015) p1

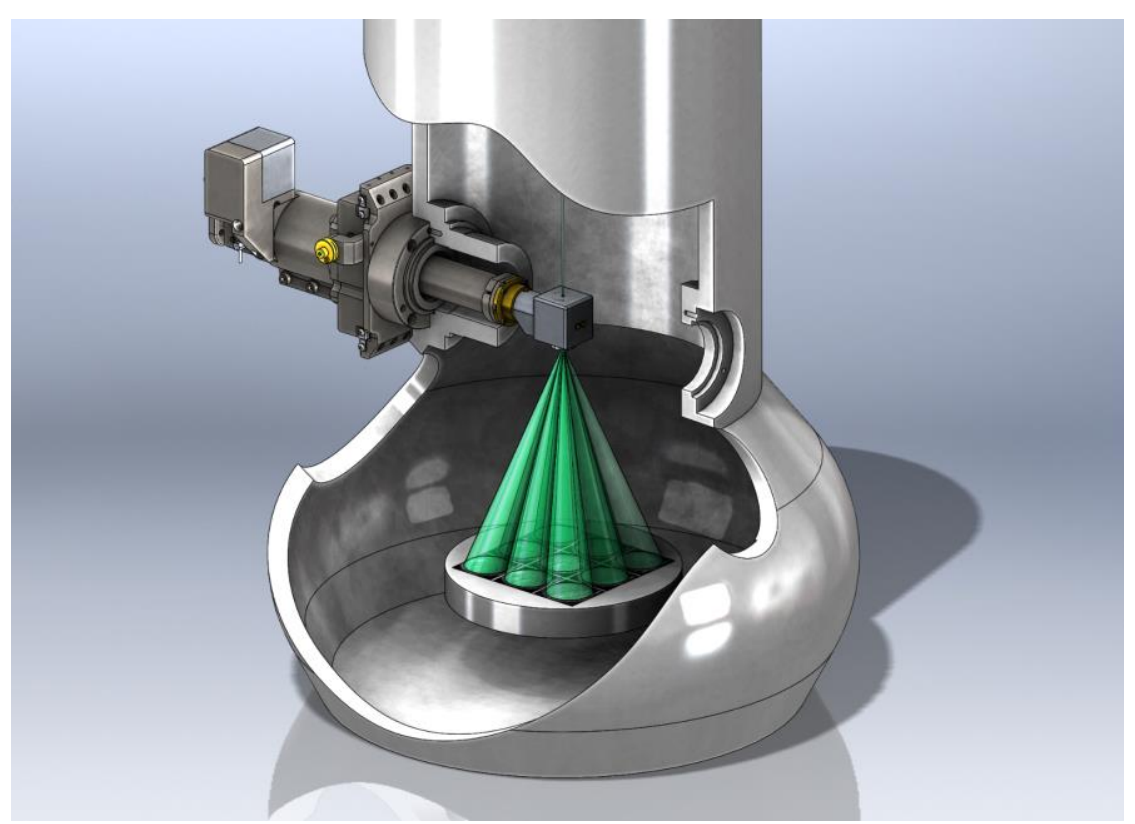

Figure 1. Schematic rendering of the TCS electrostatic deflector assembly installed in a standard TEM accessory port.

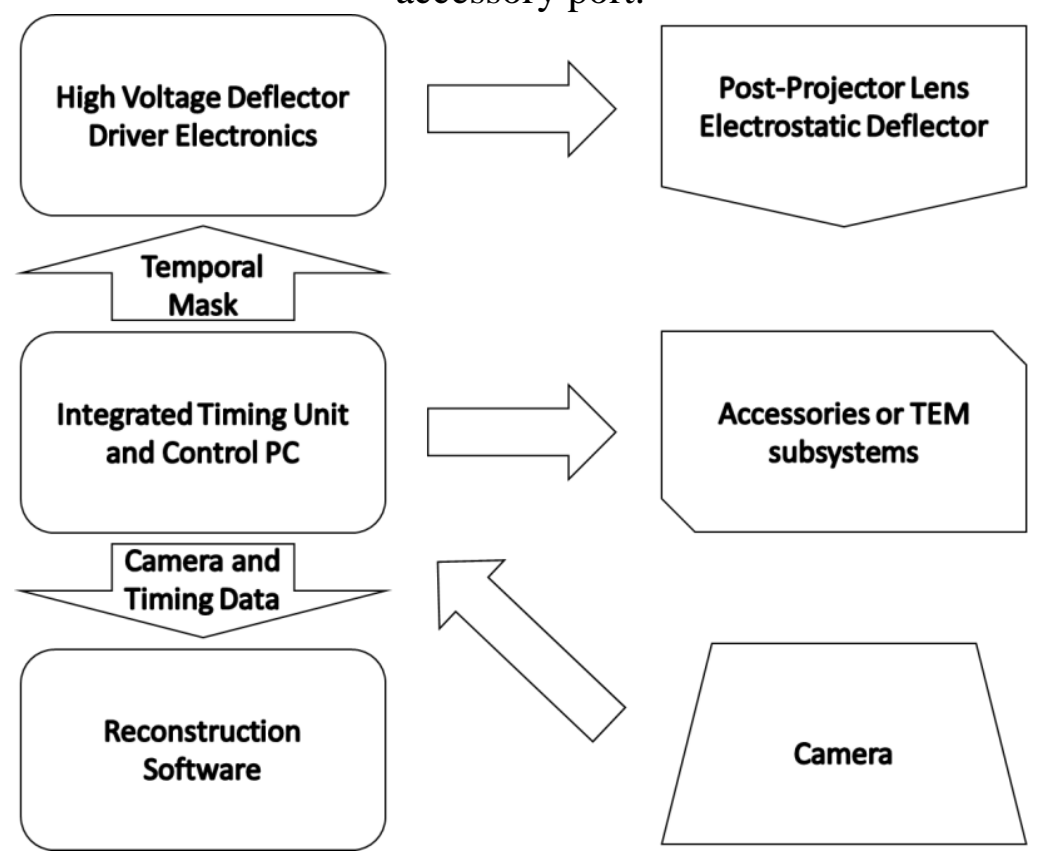

Figure 2. Block diagram of the TCS control system 